\title{
Exploration of the regulation and control mechanisms of miR-145 in trophoblast cell proliferation and invasion
}

\author{
ZHENJING CHI and MULING ZHANG \\ Department of Obstetrics, Huai'an First People's Hospital, Huai'an, Jiangsu 223300, P.R. China
}

Received August 25, 2017; Accepted January 3, 2018

DOI: $10.3892 /$ etm.2018.6890

\begin{abstract}
Preeclampsia (PE) is the leading cause of maternal and fetal mortality and morbidity. Furthermore, recent studies have reported that miR-145 within the preeclamptic trophoblast debris may cause the high blood pressure via interacting with the maternal endothelium. The aim of the present study was to investigate the functions of miR-145 in PE. Reverse transcription-quantitative polymerase chain reaction and western blotting were used to assess the expression of miR-145 and mucin (MUC1), respectively. TargetScan, miRBase and miRWalk were used to predict the targets of miR-145. Constructed miR-145 mimic plasmids were transfected into HTR-8/SVneo cells for further experiments, including an MTT assay for cell proliferation, Transwell assay for cell invasion and flow cytometry for cell apoptosis analysis. Additionally, the luciferase reporter gene system was employed for target verification. The results demonstrated that miR-145 is downregulated and MUC1 is upregulated in PE tissues and cells compared with normal placenta tissues and cells. The correlation analysis suggests that the expression of miR-145 is negatively correlated with MUC1. Meanwhile, increased proliferation, enhanced invasion and decreased apoptosis of HTR-8/SVneo cells was observed in miR-145 mimic groups compared with mimic control group. Also, the decreased luciferase activity in the miR-145 mimic group indicates that MUC1 may be a target of miR-145. In summary, the results of the present study suggest that miR-145 may serve key roles in the regulation of trophoblast cell proliferation and invasion by targeting MUC1.
\end{abstract}

\section{Introduction}

Pregnancy-induced hypertension (PIH), also known as gestational hypertension, is a pregnancy-specific condition

Correspondence to: Dr Muling Zhang, Department of Obstetrics, Huai'an First People's Hospital, 6 West Beijing Road, Huai Yin, Huai'an, Jiangsu 223300, P.R. China

E-mail: mulingzhang789@sina.com

Key words: microRNA-145, mucin 1 cell surface associated protein, preeclampsia, proliferation, invasion, target characterized by high blood pressure, edema, proteinuria and heart and kidney failure (1). PIH is defined as having a diastolic blood pressure (DBP) $>90 \mathrm{mmHg}$ and systolic blood pressure (SBP) $>140 \mathrm{mmHg}$ (2). Furthermore, PIH can be classified as mild, moderate and severe (3). Severe PIH is called preeclampsia (PE), which typically occurs after 20 weeks of gestation and may be accompanied by proteinuria, twitch and coma (3). PE is diagnosed why $\mathrm{SBP} \geq 160 \mathrm{mmHg}$ and $\mathrm{DBP} \geq 110 \mathrm{mmHg}$ (4). Previous studies have demonstrated that $\mathrm{PE}$ is a common and severe health problem for fetuses and expectant women, a leading cause of maternal and fetal mortality and morbidity (5). PE is associated with fetus maldevelopment and may lead to eclampsia and even miscarriage (5-7). Worldwide, there are $\sim 55,000$ deaths associated with PE reported annually (7), and the underlying cause of $\mathrm{PE}$ remains unknown. There is currently no effective therapeutic or preventative treatment available for PE.

Some previous studies have focused their investigations on trophoblast and placental dysfunction, which initiate pathogenesis (8). Successful implantation and placentation induces a series of cellular events associated with embryo attachment to the endometrial wall, including embryonic trophoblast cell proliferation and migration as well as invasion of the trophoblast cells into the endometrium (9). Furthermore, successful implantation is associated with remodeling of the uterine spiral via extravillous trophoblast (EVT) cell invasion (10). EVT cell invasion results in enlarged vessel diameter and the perfusion of utero placenta, which leads to increased blood flow volume and oxygen transportation (11). As such, abnormalities in artery remodeling may cause decreased blood flow volume and placental hypoxia, resulting in trophoblast dysfunction and possibly preeclampsia (12). Studying this process may increase our understanding of the onset and development of preeclampsia and potential novel treatment methods (13). It has also been reported that some signaling and genetic pathways involving vascular endothelial growth factor (VEGF), placental growth factor and transforming growth factor- $\beta$ contribute to PE development $(7,14)$. Furthermore, PE affects the innate immunity of the fetus due to increased levels of inflammatory immune cells and cytokines and decreased the regulation of immune cells and cytokines, including IL-6, certain chemokines (chemokine ligand 5) and adhesion molecules (VCAM, ICAM and E-selectin) (15).

MicroRNAs (miRNAs or miRs) are a family of small non-coding RNAs that are able to regulate gene expression via degrading mRNA at the post-translational level. Therefore, 
dysregulation of miRNAs is associated with a number of diseases (16). It has previously been reported that C19MC, miR-371-3 cluster and C14MC encode pregnancy-associated microRNAs that are responsible for the subsequent onset of gestational hypertension $(17,18)$.

It has been reported that miR-145 may be able to regulate the expression of sex determining region Y-box 2 and influence the proliferation and invasion ability of JAR and JEG-3 cells in the development of human choriocarcinomas $(18,19)$. Mucin (MUC1) is a type 1 transmembrane protein that serves a vital role in trophoblast cell proliferation and migration (20). Due to its large size, MUC1 works as a lubricant to and affects cell-cell and cell-substrate interactions, reducing cell adhesion in tumor tissues (21). Furthermore, a previous study demonstrated that MUC1 facilitates cell-cell adhesion by binding intercellular adhesion molecule-1 (ICAM-1) and E-selectin (22). The aim of the present study was to define the roles of miR-145 in trophoblast cell proliferation and invasion. In addition, the impact of MUC1 on trophoblast cell proliferation and invasion profile was explored.

\section{Materials and methods}

Patient tissues. Placenta villi tissues were obtained from 60 patients (age range, 25-35 years) who underwent caesarean section at Huai'an First People's Hospital (Huai'an, China) between April 2014 and May 2016. The tissues included 30 normal placentas (NP) as controls and 30 placentas from women with PE. The inclusion criteria were as follows: A confirmed diagnosis of PE, systolic blood pressure $>140 \mathrm{~mm} \mathrm{Hg}$ and diastolic blood pressure $>90 \mathrm{mmHg}$. Patients were compared with 30 healthy controls which were aged matched. All patients with complications of pregnancy, including, twins, fetal gene abnormalities, maternal chronic hypertension, aberrant liver enzyme levels, cardiovascular disease, renal disease, diabetes or other infectious diseases were excluded from the current study.

Fresh tissues were flash frozen in liquid nitrogen $\left(-196^{\circ} \mathrm{C}\right)$. The present study was approved by the Ethics Committee of Huai'an First People's Hospital.

Cell lines. The HTR-8/SVneo cell line was purchased from Otwo Biotech, Inc., (Shenzhen, China). and cultured in RPMI 1640 medium (Gibco; Thermo Fisher Scientific, Inc., Waltham, MA, USA) with $10 \%$ fetal bovine serum (FBS; Gibco, Thermo Fisher Scientific, Inc.) at $37^{\circ} \mathrm{C}$ in an atmosphere containing $5 \%$ $\mathrm{CO}_{2}$. The subcultivation ratio was $1: 3$ to $1: 8$ and cells were sub-cultured 2 to 3 times a week. Cultured cells at passage 4-5 were subsequently used for cell transfection, MTT, Transwell and flow cytometry assays.

MiR-145 target gene predictions. TargetScan (http://www. targetscan.org/vert_71/2, miRBase (http://www.mirbase. org/2 and miRWalk (http://zmf.umm.uni-heidelberg. de/apps/zmf/mirwalk2/) were used for miR-145 target gene prediction. MUC1 was identified as a target gene of miR-145. Pearson's correlation analysis was therefore performed for miR-145 and MUC1 in PE tissues.

Cell transfection. HTR-8/SVneo cells were seeded in 24 well-plates in the density of $5 \times 10^{3}$ cells/well and divided into three groups: The negative control group (NC), the miR-145 mimic group transfected with miR-145 overexpression plasmids (miR-145 mimic) and the miR-145 mimic control group transfected with empty plasmids (mimic control). The constructed miR-145 mimic and mimic control were purchased from Cyagen Biosciences, Inc. (Santa Clara, CA, USA). A total of 500 ng pBR322 plasmid (cat. no. 15367014; Invitrogen; Thermo Fisher Scientific, Inc.) and 2.5 $\mu$ 1 Lipofectamine ${ }^{\circledR}$ 2000 transfection reagent (11668027; Invitrogen; Thermo Fisher Scientific, Inc.) were added to each well. RPMI 1640 (Gibco; Thermo Fisher Scientific, Inc.) was added to reach a total of $1 \mathrm{ml}$ in each well. Following $6 \mathrm{~h}$ of incubation at $37^{\circ} \mathrm{C}$, the medium was replaced with fresh RPMI 1640 medium containing $10 \%$ bovine serum albumin (cat. no. A8020; Beijing Solarbio Science \& Technology Co., Ltd., Beijing, China). Following $48 \mathrm{~h}$ transfection, the expression of miR-145 and MUC1 were verified using RT-qPCR and western blotting respectively, both in the tissues and HTR-8/SVneo cells. This experiment was performed in triplicate.

Reverse transcription-quantitative polymerase chain reaction (RT-qPCR) used for miR-145 mRNA expression. RT-qPCR was employed to assess the relative expression of miR-145 in NP tissue, PE tissue, the miR-145 mimic and transfected HTR-8/SVneo cells. An RNeasy Mini Kit (Qiagen GmbH, Hilden, Germany) was utilized to isolate RNA in tissues and cells according to the manufacturer's protocol. RNA concentration was determined using a Nanodrop 2000 spectrophotometer (Thermo Fisher Scientific, Inc., Pittsburgh, PA, USA). The M-MLV 1st Strand kit (C28025032; Invitrogen; Thermo Fisher Scientific, Inc., Waltham, MA, USA) was used to synthesize cDNA by RT according to the manufacturer's protocol. A Power SYBR-Green Real-Time PCR Master Mix kit (RP014A; Takara Bio, Inc., Otsu, Japan) was used for qPCR. The temperature protocol for RT was as follows: $43^{\circ} \mathrm{C}$ for $30 \mathrm{~min}, 98^{\circ} \mathrm{C}$ for $5 \mathrm{~min}$ and $5^{\circ} \mathrm{C}$ for $5 \mathrm{~min}$. The PCR conditions were as follows: Pre-denaturation at $95^{\circ} \mathrm{C}$ for $6 \mathrm{~min}$ followed by 36 cycles of initiation at $94^{\circ} \mathrm{C}$ for $30 \mathrm{sec}$, annealing at $60^{\circ} \mathrm{C}$ for $30 \mathrm{sec}$, and elongation at $75^{\circ} \mathrm{C}$ for $90 \mathrm{sec}$. Samples were subsequently stored at $4^{\circ} \mathrm{C}$. Endogenous U6 was used as a reference gene to normalize the miR-145 expression. Human-miR-145 RT-qPCR Primer Set 200 rxn was used as the miR-145 primers, forward, 5'-GAGAACTCC AGCTGGTCCTTA-3', and reverse, 5'-GGTGGGAAGGAG GCAAAT-3' (AM30047; Applied biosystems; Thermo Fisher Scientific, Inc.) and the RT-qPCR Primer Set 200 rxn was used as U6 primers, forward, 5'-CGCTTCGGCAGCACATATACT AA-3', reverse, 5'-TATGGAACGCTTCACGAATTTGC-3' (AM30303; Boyao Biotechnology, Shanghai, China). This experiment was performed in triplicate. The expression of miR-145 was determined using the $2^{-\Delta \Delta C q}$ method (23).

Western blotting for MUC1 protein expression. Western blotting was performed to identify the expression of MUC1 in PE tissues and HTR-8/SVneo cell transfected with the miR-145 mimic. Tissues or Cells were lysed using radio immunoprecipitation assay buffer (P0013B, Beyotime Institute of Biotechnology, Haimen, China) and protein was quantified using a bicinchoninic acid assay kit (23227; Beijing Biotides Biotechnology Co., Ltd., Beijing, China). GAPDH was used 
as an internal reference. Protein $(30 \mu \mathrm{g})$ in tissues or cells was separated by $12 \%$ SDS-PAGE and transferred onto polyvinylidene fluoride membranes (Thermo Fisher Scientific, Inc.). Membranes were incubated with antibodies against MUC1 (1:1,000; cat. no. PA525939; Thermo Fisher Scientific, Inc.) and GAPDH (1:500; cat. no. PA1987; Thermo Fisher Scientific, Inc.) for $1 \mathrm{~h}$ at $25^{\circ} \mathrm{C}$. Membranes were blocked with $5 \%$ bovine serum albumin (Beijing Solarbio Science \& Technology Co., Ltd.) for $1 \mathrm{~h}$ at $25^{\circ} \mathrm{C}$. Membranes were subsequently incubated for $45 \mathrm{~min}$ at $25^{\circ} \mathrm{C}$ with the goat anti-rabbit IgG (H+L) Highly Cross-Adsorbed secondary antibody, Alexa Fluor ${ }^{\circledR}$ Plus 800 (1:20,000; cat. no. A32735, Thermo Fisher Scientific, Inc.). An enhanced chemiluminescence (ECL) western blotting kit (32209; Thermo Fisher Scientific, Inc.) was used to visualize the bands. Gray values were obtained using ImageJ software version 1.51j8 (National Institutes of Health, Bethesda, MD, USA). This experiment was performed in triplicate.

MTT assay for HTR-8/SVneo cell proliferation. An MTT assay was performed to assess cell proliferation. A total of 2,000 transfected HTR-8/SVneo cells were seeded in 24-well plates and incubated at $37^{\circ} \mathrm{C}$ for $4 \mathrm{~h}$ in an atmosphere containing $5 \%$ $\mathrm{CO}_{2}$. Subsequently, an MTT Cell Viability Assay kit (KA1606; Abnova Corporation, Taipei, Taiwan) was used according to the manufacturer's protocol. The optical density at $570 \mathrm{~nm}$ wavelength was detected using microplate reader at 24, 48 and $72 \mathrm{~h}$ following transfection. This experiment was conducted in triplicate.

Matrigel assay for HTR-8/SVneo cell invasion. Transwell culture inserts (8-mm pore size; Falcon; BD Biosciences, Franklin Lakes, NJ, USA) were placed into the wells of 24-well culture plates with separated the upper and lower chambers. Matrigel (BD Biosciences) was pre-coated on the upper side of the membrane and incubated at $37^{\circ} \mathrm{C}$ for $1 \mathrm{~h}$ for gel formation. The membrane was hydrated in FBS for $2 \mathrm{~h}$ prior to use. In the lower chamber, $600 \mu 1$ RPMI 1640 (Thermo Fisher Scientific, Inc.) containing $10 \% \mathrm{FBS}$ was added and $1 \times 10^{5}$ cells/well were seeded to the upper chamber. Cells in inserts were fixed using $4 \%$ paraformaldehyde at $4^{\circ} \mathrm{C}$ for $15 \mathrm{~min}$ and stained with $0.05 \%$ crystal violet for $10 \mathrm{~min}$ at room temperature. Cells were counted under a light microscope (magnification, $\mathrm{x} 200$ ) following $72 \mathrm{~h}$ incubation at $37^{\circ} \mathrm{C}$.

Flow cytometry assay for HTR-8/SVneo cell apoptosis. Following transfection, a flow cytometry assay was performed to study the apoptosis of HTR-8/SVneo cells. Briefly, the transfected cells $\left(1 \times 10^{5}\right.$ cells/well) were seeded in a 24 -well plate and incubated at $37^{\circ} \mathrm{C}$ for $72 \mathrm{~h}$. HTR-8/SVneo cells were stained with propidium iodide and Annexin V-fluorescein isothiocyanate (BD Biosciences) in the dark for $15 \mathrm{~min}$ at room temperature. Stained cells were subsequently examined using a FACScan laser flow cytometer (BD Biosciences) equipped with CellQuest software version 3.1 (BD Biosciences).

Luciferase reporter gene analysis for target verification. For the luciferase reporter assay, HTR-8/SVneo cells (2,500 cells/well) were seeded in 96 -well plates and incubated at $37^{\circ} \mathrm{C}$ in an atmosphere containing $5 \% \mathrm{CO}_{2}$ for $24 \mathrm{~h}$. Subsequently, MUC1-3'UTR-wild type (WT) and MUC1-3'UTR-mutant
(MUT) plasmids (2.5 $\mu \mathrm{g}$; Cyagen Biosciences, Inc.) were transfected into the NC, miR-145 mimic and mimic control groups using Lipofectamine ${ }^{\circledR}$ 2000. Then, a Luciferase Reporter Assay kit (K801-200; Wuhan Amyjet Scientific Co., Ltd., Wuhan, China) was used according to the manufacturer's instruction.

Statistical analysis. All data are presented as the mean \pm standard deviation. Data were analyzed using GraphPad Prism version 5.01 (GraphPad Software, La Jolla, CA, USA). Student's t test and one-way analysis of variance followed a Dunnett's post hoc test were employed for data comparisons. $\mathrm{P}<0.05$ was considered to indicate a statistically significant difference.

\section{Results}

Relative expression of miR-145 mRNA and MUC1 protein in $N P$ and PE tissues. To investigate the expression profile of miR-145 mRNA and MUC1 protein in normal placental and the PE placental tissues, RT-qPCR and western blotting were performed, respectively. miR-145 was significantly downregulated and MUC1 was significantly upregulated in PE compared with NP tissues $(\mathrm{P}<0.01$; Fig. 1).

Correlation analysis between miR-145 and MUC1 expression. Correlation analysis was performed to investigate the association between miR-145 and MUC1 expression level. The results revealed that the expression of MUC1 was negatively correlated with miR-145 expression ( $r=0.9728$; Fig. 2).

Relative expression of MUC1 in the miR-145 mimic group. Following transfection of miR-145 mimic plasmids, the expression of miR-145 and the MUC1 were determined by RT-qPCR and western blotting respectively. miR-145 expression was significantly higher $(\mathrm{P}<0.01)$ and MUC1 expression was markedly downregulated in the miR-145 mimic group compared with the NC group (Fig. 3).

MTT assay for HTR-8/SVneo cell proliferation determination. To explore the proliferation of HTR-8/SVneo cells, and MTT assay was performed. HTR-8/SVneo cells were constructed miR-145 mimic plasmids and mimic control plasmids. Results demonstrated that the cell proliferation rate was significantly increased in the miR-145 mimic group at $72 \mathrm{~h}$ following transfection compared with the mimic control group $(\mathrm{P}<0.01$; Fig. 4). This result suggested that miR-145 may therefore be able to promote cell proliferation.

Transwell assay for HTR-8/SVneo cell invasion determination. The effects of miR-145 on HTR-8/SVneo cell invasion ability were assessed using a Transwell assay. Cell invasion was markedly increased in the miR-145 mimic group compared with the mimic control group (Fig. 5). Hence, miR-145 may contribute to the invasion ability of HTR-8/SVneo cells.

Flow cytometry assay for HTR-8/SVneo cell apoptosis. Flow cytometry was performed at $72 \mathrm{~h}$ following plasmid transfection. Subsequently, HTR-8/SVneo cells were stained with propidium iodide and Annexin $\mathrm{V}$-fluorescein isothiocyanate. 


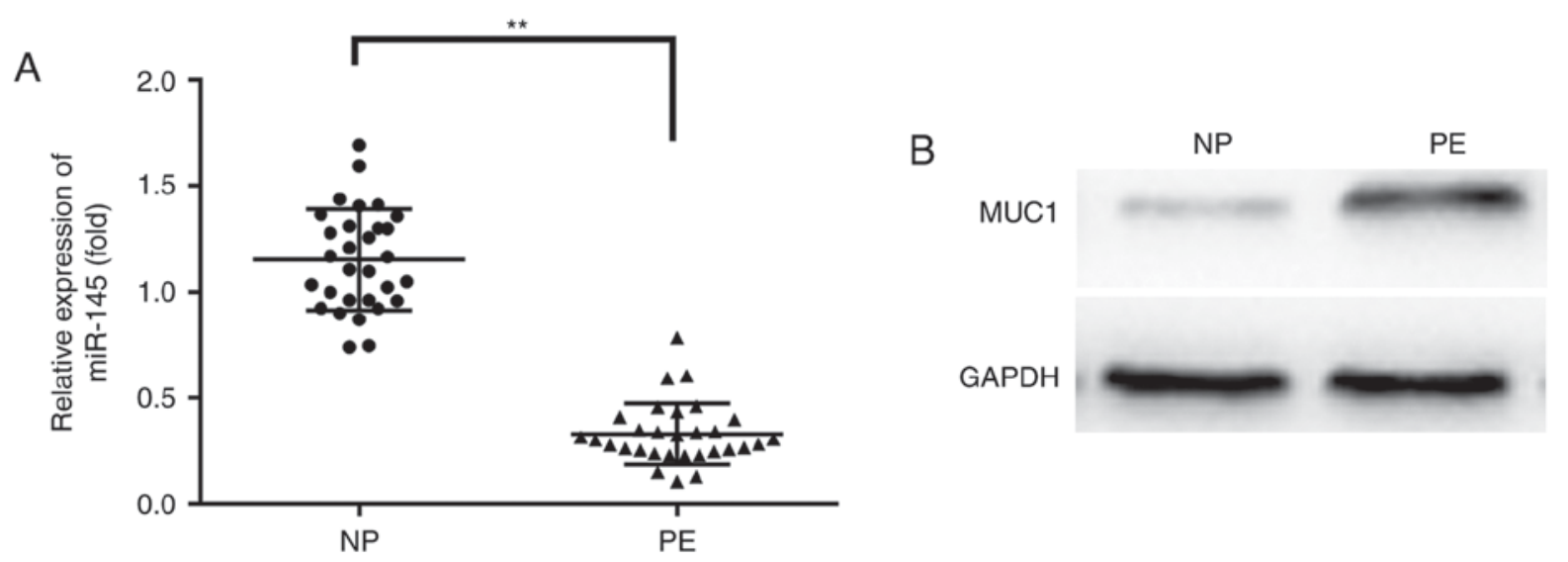

Figure 1. Relative expression profile of miR-145 and MUC1 in NP and PE tissues (A) Relative expression of (A) miR-145 and (B) MUC1 in NP and PE tissues. ${ }^{* *} \mathrm{P}<0.01$. miR, microRNA; MUC1, mucin; NP, normal placental; PE, preeclampsia.

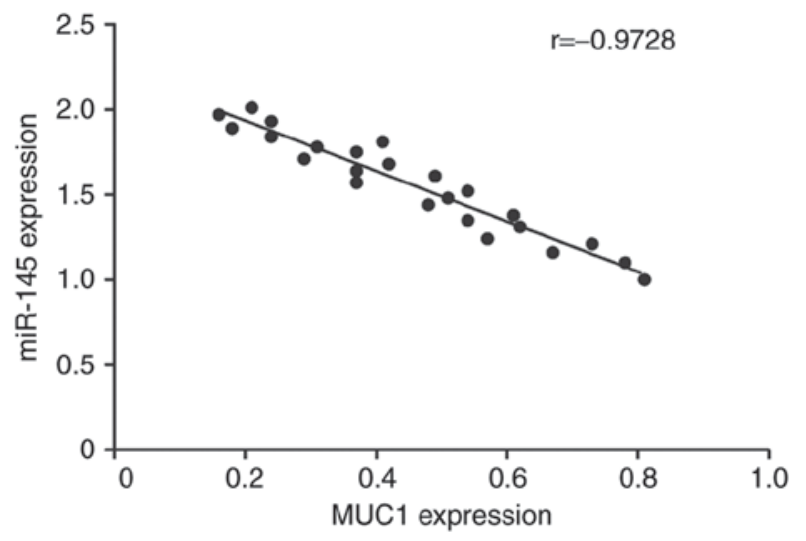

Figure 2. Correlation between miR-145 and MUC1 expression in $24 \mathrm{NP}$ tissue samples. $r=-0.9728$. miR, microRNA; MUC1, mucin; NP, normal placental.

A

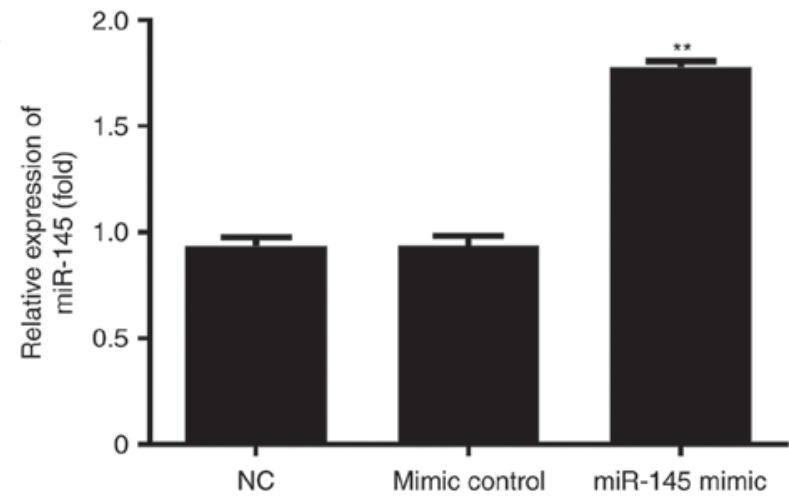

B

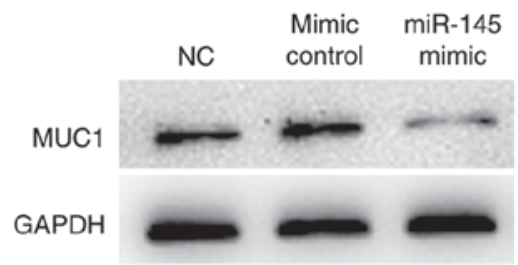

Figure 3. Expression of MUC1 in the miR-145 mimic group. Expression of (A) miR-145 as determined by reverse transcription-quantitative polymerase chain reaction and (B) MUC1 as assessed using western blotting. ${ }^{* *} \mathrm{P}<0.01$. MUC1, mucin; miR, microRNA; NC, negative control.

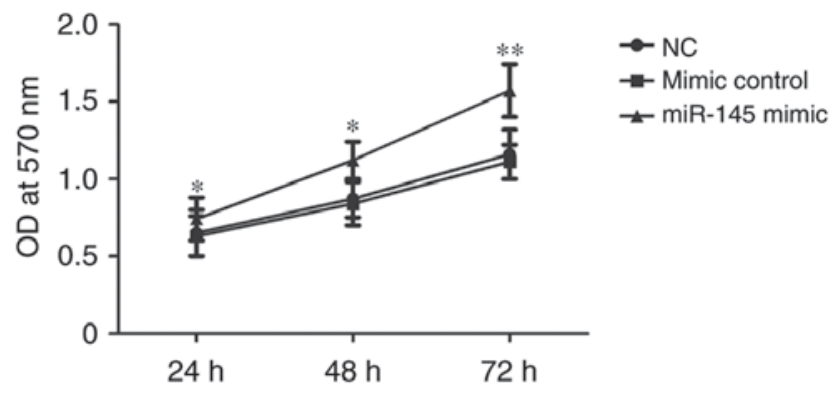

Figure $4 . \mathrm{OD}$ at $570 \mathrm{~nm}$ was detected at 24,48 and $72 \mathrm{~h}$ following transfection in the NC, mimic control and miR-145 mimic group. ${ }^{*} \mathrm{P}<0.05$ and ${ }^{* *} \mathrm{P}<0.01$ vs. NC and mimic control groups. OD, optical density; NC, negative control; miR, microRNA.

The results indicated that apoptosis was significantly decreased in the miR-145 mimic group compared with the mimic control group and the mimic control was not significantly different to the NC $(\mathrm{P}<0.05$; Fig. 6$)$.

Luciferase reporter gene analysis for target verification. The luciferase reporter system was used to verify that MUC1 was a target gene of miR-145 in HTR-8/SVneo cells. Firstly, the database was searched for target prediction. The results revealed that MUC1 was a putative target gene of miR-145 (Fig. 7A). The constructed MUC1 mutant and wild type plasmids were co-transfected with miR-145 mimic and mimic control plasmids into HTR-8/SVneo cells. The fluorescence intensity and results of western blotting were significantly lower in the miR-145 mimic and MUC1-3'UTR-WT group compared with the mimic control group $(\mathrm{P}<0.01$, Fig. 7A; $\mathrm{P}<0.001$, Fig. 7B). There were no significant difference between mimic control and NC. Therefore, MUC1 confirmed as a target gene of miR-145 in HTR-8/SVneo cells.

\section{Discussion}

It has previously been demonstrated that miRNA serve vital roles in cell proliferation, migration, invasion and apoptosis processes in various cancers (24). It is well known that miRNAs are able to combine to the putative 3'-UTR of mRNAs to alter 


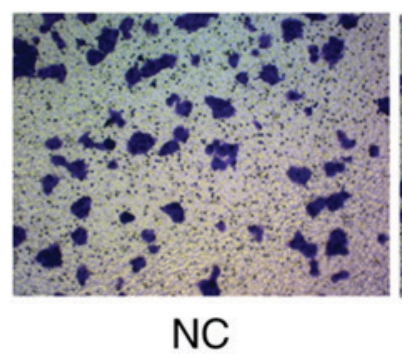

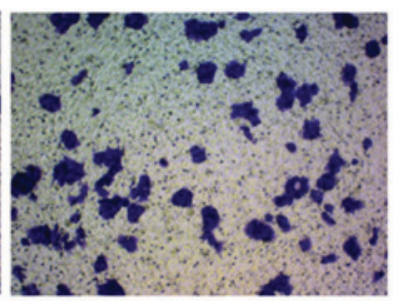

Mimic control

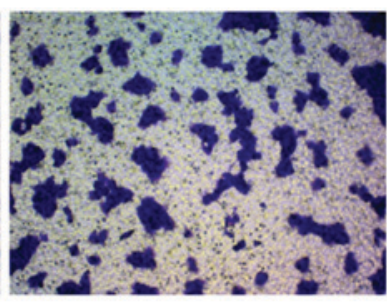

miR-145 mimic

Figure 5. Invasion activity in the NC, mimic control and miR-145 mimic groups. Cells were stained with $0.05 \%$ crystal violet and transwell assays were performed $72 \mathrm{~h}$ post-transfection. Magnification, x200. NC, negative control; miR, microRNA.
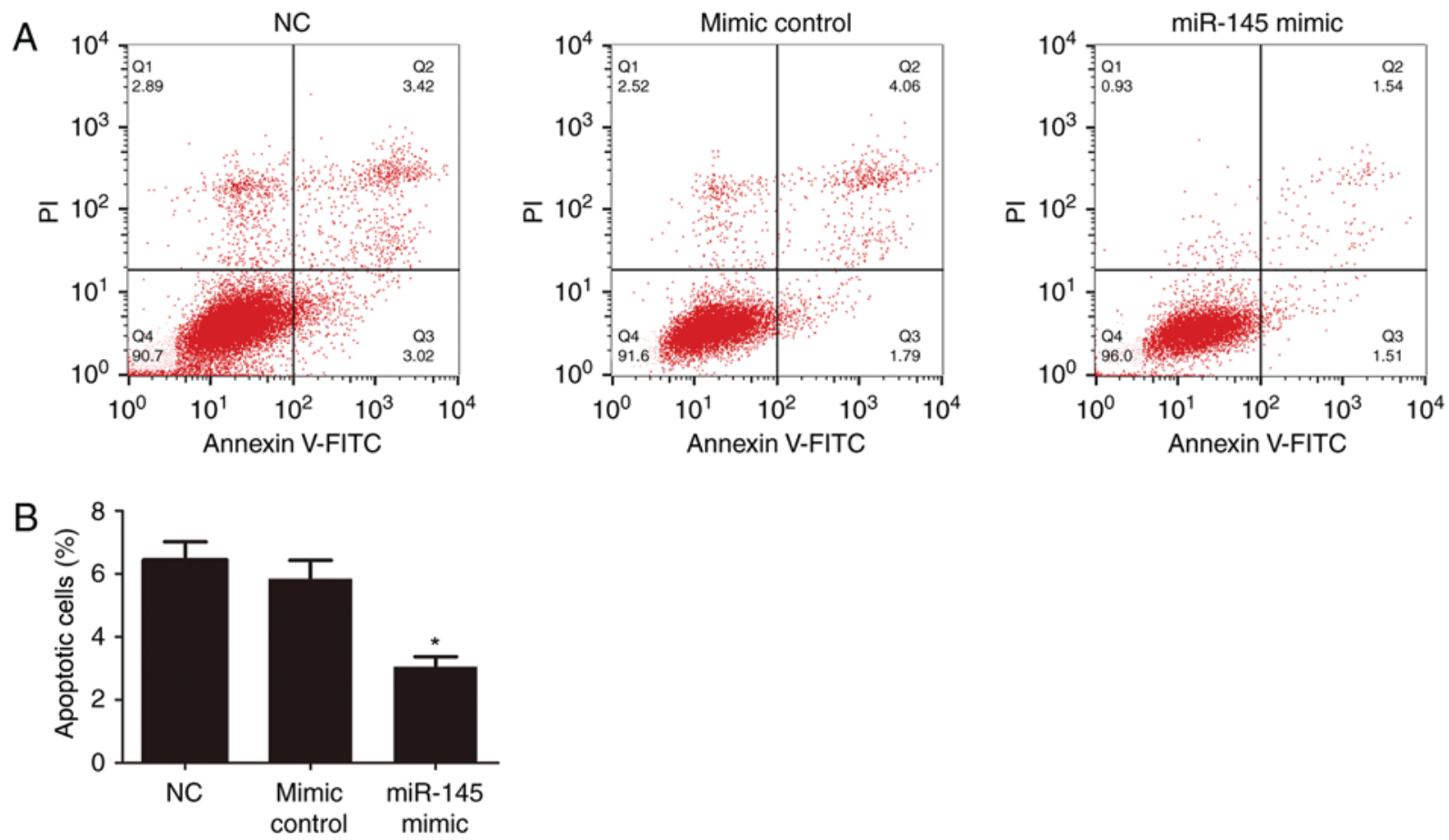

Figure 6. Apoptosis profile in the NC, mimic control and miR-145 mimic groups. Cells were stained with Annexin V-FITC and PI and assessed using flow cytometry. (A) Apoptosis distribution and (B) percentage of apoptotic cells in the NC, mimic control and miR-145 mimic group. NC, negative control; miR, microRNA; FITC, fluorescein isothiocyanate; PI, propidium iodide. ${ }^{*} \mathrm{P}<0.05$ vs. mimic control and NC.
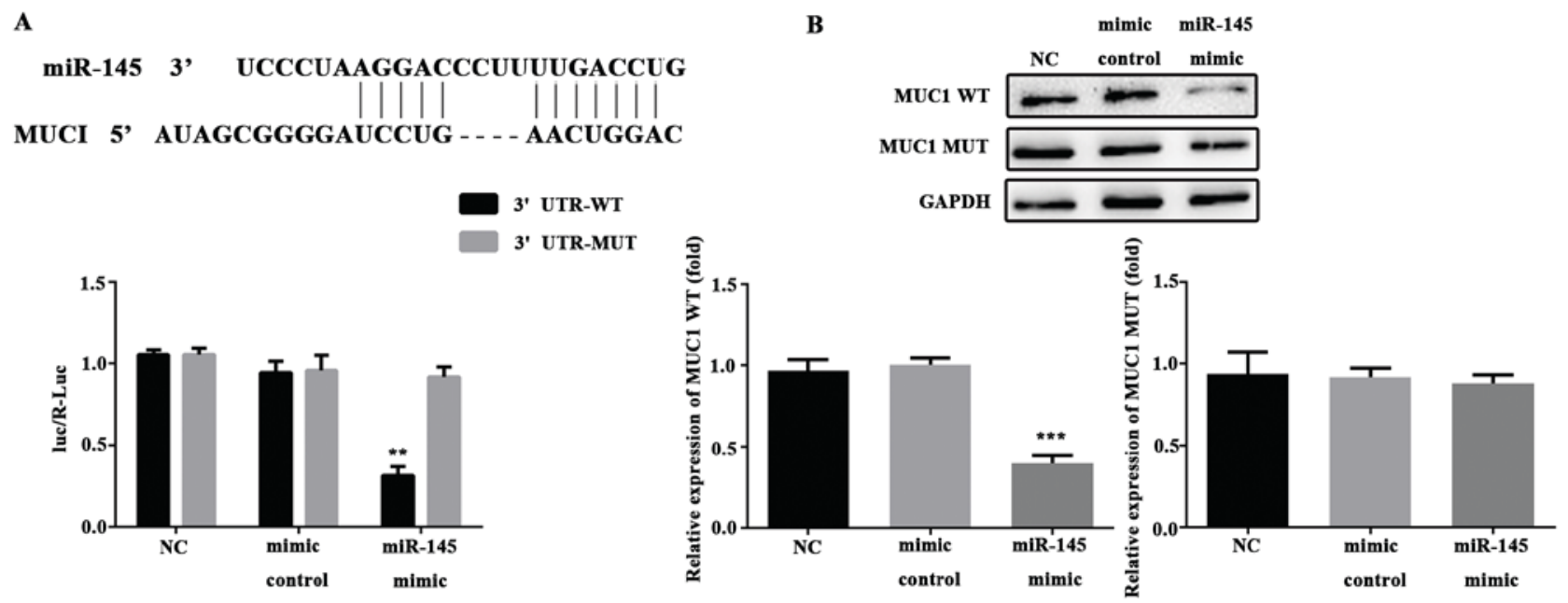

Figure 7. Luciferase reporter assay for target verification in the NC, mimic control and miR-145 mimic groups. (A) Gene target prediction obtained using bioinformatics software. Luciferase activity in NC, miR-145 mimic and mimic control groups. ${ }^{* *} \mathrm{P}<0.01$ vs. mimic control and NC. (B) Western blotting was performed to assess MUC1 expression. Grey value for MUC1 expression in the MUC1 MUT and MUC1 WT groups ${ }^{* * *} \mathrm{P}<0.001$ vs. mimic control and NC. NC, negative control; miR, microRNA; MUC1, mucin; MUT, mutant; WT, wild type; UTR, untranslated region. 
the function of various genes at the post-translational level, and so it was hypothesized that miRNAs may function as a regulator of some gene that is associated with the development of placenta $(25,26)$. In addition, the underlying mechanisms of miRNAs in placenta have not been fully explored (27). In the present study, miR-145 was used as the target and the results demonstrated that miR-145 was downregulated in PE tissues compared with NP tissues. Furthermore, MUC1 was identified as a target gene of miR-145 via gene prediction software and the luciferase reporter assay. In addition, MTT, Transwell and flow cytometry assays was performed in HTR-8/SVneo cells. Further investigation revealed that miR-145 may promote HTR-8/SVneo cell proliferation and invasion whilst suppressing cell apoptosis. Therefore, in the present study, the roles of miR-145 in placenta development were identified and its potential target MUC1 was explored.

It has previously been reported that miR-145 functions differently in various types of cancer (28). For example, miR-145 inhibits cell growth in gallbladder cancer (29). Suppressed cell migration and cancer invasion has also been reported, which suggests that miR-145 may function as a tumor suppressor (30). The results of the present study are in accordance with those of a previous study that indicated that miR-145 is a positive regulator of endogenously regulated trophoblast expansion (19). Genes associated with trophoblast invasion include heme oxygenase-1, epidermal growth factor (EGF), VEGF and MUC1 $(21,31,32)$. The results of the present study and previous research indicate that there are two pathways of MUC1 in cancer cell-cell interactions; in tumor cells, MUC1 facilitates metastasis and inhibits cell-cell adhesion by binding to ICAM-1 and E-selectin (21), whereas in the female reproductive tract $\mathrm{MUC1}$ overexpression reduced the invasion capacity of HTR-8/SVneo cells (20). The primary aim of the present study was to combine miR-145 and MUC1 to investigate the effect of miR-145 in trophoblast cells and confirm MUC1 as a target of miR-145.

The present study has several limitations due to the experimental design. Although it was specified that MUC1 was a target gene of miR-145 in HTR-8/SVneo cells, the underlying mechanism remains to be elucidated. Future studies should be performed to explore the whole signaling pathway associated with miR-145 and MUC1 including the downstream genes associated with cell proliferation, invasion and apoptosis. For instance, the B-cell lymphoma 2/B-cell lymphoma 2-associated $\mathrm{X}$ protein signaling pathway, which is a target of $\mathrm{MUC1}$, is associated with cell apoptosis (33). Furthermore, proteins that serve roles in cell adhesion, including ICAM-1 and E-selectin, may be used to assess cell migration and invasion (21). Adrenomedullin 2 (ADM2) has been reported to be associated with spontaneous abortion in humans and inhibits the expression of MUCI (34). Serum ADM2 is downregulated in PE, suggesting that decreased levels of ADM2 level may induce the overexpression of MUC1 (34). In future investigations, multiple factors that regulate the expression of MUC1 should be explored.

In summary, miR-145 serves an important role in the development of trophoblast cells. The results indicated that the proliferation was promoted and invasion was inhibited by miR-145 targeting MUC1. Therefore, miR-145 may serve a role in the development of PE via its effect on trophoblast cells.

\section{Acknowledgements}

Not applicable.

\section{Funding}

No funding was received.

\section{Availability of data and materials}

The analyzed data sets generated during the present study are available from the corresponding author on reasonable request.

\section{Authors' contributions}

ZC and MZ conceived and designed the experiments and analyzed the data. ZC performed the experiments and contributed to the reagents/materials/analysis tools.

\section{Ethics approval and consent to participate}

The present study was approved by the Ethics Committee of Huai'an First People's Hospital and written informed consent was obtained from each patient prior to the study.

\section{Patient consent for publication}

Not applicable.

\section{Competing interests}

The authors declare that they have no competing interests.

\section{References}

1. Mei Z, Huang B, Mo Y and Fan J: An exploratory study into the role of miR-204-5p in pregnancy-induced hypertension. Exp Ther Med 13: 1711-1718, 2017.

2. George EM and Granger JP: Endothelin: Key mediator of hypertension in preeclampsia. Am J Hypertens 24: 964-969, 2011.

3. Kintiraki E, Papakatsika S, Kotronis G, Goulis DG and Kotsis V: Pregnancy-induced hypertension. Hormones (Athens) 14: 211-223, 2015.

4. Obed $\mathrm{S}$ and Patience A: Birth weight and ponderal index in pre-eclampsia: A comparative study. Ghana Med J 40: 8-13, 2006.

5. Choudhury M and Friedman JE: Epigenetics and microRNAs in preeclampsia. Clin Exp Hypertens 34: 334-341. 2012.

6. Sheikh AM, Small HY, Currie G and Delles C: Systematic review of micro-RNA expression in pre-eclampsia identifies a number of common pathways associated with the disease. PLoS One 11: e0160808, 2016.

7. Gathiram P and Moodley J: Pre-eclampsia: Its pathogenesis and pathophysiolgy. Cardiovasc J Afr 27: 71-78, 2016.

8. Conrad KP and Benyo DF: Placental cytokines and the pathogenesis of preeclampsia. Am J Reprod Immunol 37: 240-249, 1997.

9. Renaud SJ, Kubota K, Rumi MAK and Soares MJ: The FOS transcription factor family differentially controls trophoblast migration and invasion. J Biol Chem 289: 5025-5039, 2014.

10. Wallace AE, Fraser R and Cartwright JE: Extravillous trophoblast and decidual natural killer cells: A remodelling partnership. Hum Reprod Update 18: 458-471, 2012.

11. Hazan AD, Smith SD, Jones RL, Whittle W, Lye SJ and Dunk CE: Vascular-leukocyte interactions : Mechanisms of human decidual spiral artery remodeling in vitro. Am J Pathol 177: 1017-1030. 2010.

12. Burke SD and Karumanchi SA: Spiral artery remodeling in preeclampsia revisited. Hypertension 62: 1013-1014, 2013. 
13. Anton L, Olarerin-George AO, Schwartz N, Srinivas S, Bastek J, Hogenesch JB and Elovitz MA: miR-210 inhibits trophoblast invasion and is a serum biomarker for preeclampsia. Am J Pathol 183: 1437-1445, 2013.

14. Craici IM, Wagner SJ, Weissgerber TL, Grande JP and Garovic VD: Advances in the pathophysiology of pre-eclampsia and related podocyte injury. Kidney Int 86: 275-285, 2014.

15. Bounds KR, Newell-Rogers MK and Mitchell BM: Four pathways involving innate immunity in the pathogenesis of preeclampsia. Front Cardiovasc Med 2: 20, 2015.

16. Gong H, Liu CM, Liu DP and Liang CC: The role of small RNAs in human diseases: Potential troublemaker and therapeutic tool. Med Res Rev 25: 361-381, 2005.

17. Hromadnikova I, Kotlabova K, Hympanova L, Doucha J and Krofta L: First trimester screening of circulating C19MC microRNAs can predict subsequent onset of gestational hypertension. PLoS One 9: e113735, 2014.

18. Xu F, Wang H, Zhang X, Liu T and Liu Z: Cell proliferation and invasion ability of human choriocarcinoma cells lessened due to inhibition of Sox 2 expression by microRNA-145. Exp Ther Med 5: 77-84, 2013.

19. Farrokhnia F, Aplin JD, Westwood M and Forbes K: MicroRNA regulation of mitogenic signaling networks in the human placenta. J Biol Chem 289: 30404-30416, 2014

20. Bojić-Trbojević Ž, Jovanović Krivokuća M, Kolundžić N Kadoya T, Radojčić L and Vićovac L: Interaction of extravillous trophoblast galectin-1 and mucin(s)-Is there a functional relevance? Cell Adh Migr 10: 179-188, 2016.

21. Thirkill TL, Cao T, Stout M, Blankenship TN, Barakat A and Douglas GC: MUC1 is involved in trophoblast transendothelial migration. Biochim Biophys Acta 1773: 1007-1014, 2007.

22. Geng Y, Yeh K, Takatani T and King MR: Three to Tango: MUC1 as a ligand for both E-selectin and ICAM-1 in the breast cancer metastatic cascade. Front Oncol 2: 76, 2012.

23. Livak KJ and Schmittgen TD: Analysis of relative gene expression data using real-time quantitative PCR and the 2(-Delta Delta C(T) method. Methods 25: 402-408, 2001.

24. Lan H, Lu H, Wang X and Jin H: MicroRNAs as potential biomarkers in cancer: Opportunities and challenges. Biomed Res Int 2015: 125094, 2015 .
25. Ito M, Sferruzzi-Perri AN, Edwards CA, Adalsteinsson BT, Allen SE, Loo TH, Kitazawa M, Kaneko-Ishino T, Ishino F, Stewart CL and Ferguson-Smith AC: A trans-homologue interaction between reciprocally imprinted miR-127 and Rt11 regulates placenta development. Development 142: 2425-2430, 2015.

26. Paikari A, D Belair C and Blelloch R: The eutheria-specific miR-290 cluster modulates placental growth and maternal-fetal transport. Development 144: 3731-3743, 2017.

27. Ouyang Y, Mouillet JF, Coyne CB and Sadovsky Y: Review: Placenta-specific microRNAs in exosomes-good things come in nano-packages. Placenta 35 (Suppl): S69-S73, 2014.

28. Iio A, Nakagawa Y, Hirata I, Naoe T and Akao Y: Identification of non-coding RNAs embracing microRNA-143/145 cluster. Mol Cancer 9: 136, 2010.

29. Letelier P, García P, Leal P, Álvarez H, Ili C, López J, Castillo J, Brebi $\mathrm{P}$ and Roa JC: miR-1 and miR-145 act as tumor suppressor microRNAs in gallbladder cancer. Int J Clin Exp Pathol 7: 1849-1867, 2014

30. Dong R, Liu X, Zhang Q, Jiang Z, Li Y, Wei Y, Li Y, Yang Q, Liu J, Wei JJ, et al: miR-145 inhibits tumor growth and metastasis by targeting metadherin in high-grade serous ovarian carcinoma. Oncotarget 5: 10816-10829, 2014.

31. Bilban M, Haslinger P, Prast J, Klinglmüller F, Woelfel T, Haider S, Sachs A, Otterbein LE, Desoye G, Hiden U, et al: Identification of novel trophoblast invasion-related genes: Heme oxygenase-1 controls motility via peroxisome proliferator-activated receptor gamma. Endocrinology 150: 1000-1013, 2009.

32. Knöfler M and Pollheimer J: IFPA award in placentology lecture: Molecular regulation of human trophoblast invasion. Placenta 33 (Suppl): S55-S62, 2012.

33. Nath S and Mukherjee P: Muc1: A multifaceted oncoprotein with a key role in cancer progression. Trends Mol Med 20: 332-342, 2014.

34. Chauhan M, Balakrishnan M, Chan R and Yallampalli C: Adrenomedullin 2 (ADM2) regulates Mucin 1 at the maternal-fetal interface in human pregnancy. Biol Reprod 93: 136, 2015. 